\title{
Using Dual-Tracer PET to Predict the Biologic Behavior of Human Colorectal Cancer
}

\author{
Wang Hui*1, Zhang Jinming*1 ${ }^{1}$, Tian Jiahe ${ }^{1}$, Qu Baolin ${ }^{2}$, Li Tianran $^{1}$, Chen Yingmao ${ }^{1}$, Liu Jian ${ }^{1}$, and Wang Shan*3 \\ ${ }^{I}$ Nuclear Medicine Department, General Hospital of the Chinese People's Liberation Army and Military Medical Postgraduate \\ College, Beijing, China; ${ }^{2}$ Department of Radiation Oncology, General Hospital of the Chinese People's Liberation Army and \\ Military Medical Postgraduate College, Beijing, China; and ${ }^{3}$ Medical Experiment and Analysis Center, General Hospital of the \\ Chinese People's Liberation Army and Military Medical Postgraduate College, Beijing, China
}

\begin{abstract}
${ }^{18} \mathrm{~F}-\mathrm{FDG}$ and $3^{\prime}$-deoxy-3'-18 F-fluorothymidine ( $\left.{ }^{18} \mathrm{~F}-\mathrm{FLT}\right)$ have been proven useful in diagnosing and staging many types of cancer but with emphasis on different aspects of tumor biology. The aim of the current study was to evaluate whether ${ }^{18} \mathrm{~F}-\mathrm{FDG}$ and ${ }^{18} \mathrm{~F}-\mathrm{FLT}$ can be used complementarily in monitoring the biologic characteristics of colorectal cancer (CRC). Methods: Human CRC cell lines SW480 and SW620 of the same genetic origin but different metastatic potential were cultured and implanted into nude mice to create CRC models. Uptake of ${ }^{18} \mathrm{~F}-\mathrm{FDG}$ and ${ }^{18} \mathrm{~F}-\mathrm{FLT}$ in SW480 and SW620 cells in vitro was assessed after incubation with radiotracers for $0,30,60,90$, and $120 \mathrm{~min}$. In vivo imaging of SW480 and SW620 tumor-bearing mice was performed using small-animal PET/CT at 60 min after injection of each tracer. A region of interest was drawn over tumor and background to calculate the tumor-to-nontumor ratio (T/NT) using software on reconstructed images. Tumor growth rate, metastatic status, and survival time were assessed in tumor-bearing mice. The relationship between uptake of the tracers, metastatic capability, and tumor marker expression was evaluated using linear regression. Results: SW480 tumors grew more quickly than SW620 tumors ( $t=3.332, P=0.004)$. A higher incidence of lung and liver metastases was noted for SW480 than for SW620 $(P=0.023)$. Uptake in SW480 and SW620 cells was significantly different between ${ }^{18} \mathrm{~F}-\mathrm{FDG}$ and ${ }^{18} \mathrm{~F}-\mathrm{FLT}(t=2.507, P=0.021$, vs. $t=3.497, P=0.002)$. In the small-animal PET study, the T/NT of ${ }^{18}$ F-FDG did not differ between SW480 and SW620 tumors ( $2.69 \pm 0.98$ vs. $3.09 \pm 1.26, P=0.524)$, but the T/NT of ${ }^{18} \mathrm{~F}-\mathrm{FLT}$ differed significantly between SW480 and SW620 tumors (3.65 \pm 0.51 vs. $2.22 \pm 0.42, P<0.001$ ). Heat shock protein 27 (HSP27) expression and integrin $\beta_{3}$ expression were higher, whereas vascular endothelial growth factor receptor 2 (VEGFR2) expression and Ki67 expression were lower, in SW480 cells than in SW620 cells. For SW480, metastases in lung and liver correlated significantly with ${ }^{18} \mathrm{~F}$-FLT uptake in tumors $(r=0.763, P=$ 0.005) and with expression of HSP27 ( $r=0.894, P=0.008)$ and integrin $\beta_{3}(r=0.635, P=0.088)$. A correlation was also found between ${ }^{18} \mathrm{~F}-\mathrm{FLT}$ uptake and expression of HSP27 $(r=$
\end{abstract}

Received Mar. 13, 2009; revision accepted Jul. 21, 2009.

For correspondence or reprints contact: Tian Jiahe, Nuclear Medicine Department, General Hospital of the Chinese People's Liberation Army and Military Medical Postgraduate College, Fuxing Rd. 28, Beijing, 100853, China.

E-mail: tianjh@vip.sina.com

${ }^{*}$ Contributed equally to this work.

COPYRIGHT @ 2009 by the Society of Nuclear Medicine, Inc.
$0.924, P=0.004)$ and integrin $\beta_{3}(r=0.813, P=0.025)$. No correlation was found between ${ }^{18} \mathrm{~F}-\mathrm{FDG}$ uptake in tumors and metastasis in lung and liver ( $r=-0.111, P=0.388)$. However, there was a significantly negative correlation between ${ }^{18} \mathrm{~F}-\mathrm{FDG}$ uptake and the survival time of tumor-bearing mice $(r=-0.500$, $P=0.017)$, to which ${ }^{18} \mathrm{~F}-\mathrm{FLT}$ did not relate $(r=0.262, P=$ 0.182). Conclusion: High uptake of ${ }^{18} \mathrm{~F}-\mathrm{FDG}$ and ${ }^{18} \mathrm{~F}-\mathrm{FLT}$ may reflect poorer survival and a higher metastatic potential for CRC in mice. Combining ${ }^{18} \mathrm{~F}-\mathrm{FDG}$ with ${ }^{18} \mathrm{~F}$-FLT PET would be helpful in better predicting the biologic behavior of $\mathrm{CRC}$.

Key Words: ${ }^{18} \mathrm{~F}-\mathrm{FDG}$; ${ }^{18} \mathrm{~F}-\mathrm{FLT}$; biological behavior; positron emission tomography; colorectal cancer

J Nucl Med 2009; 50:1857-1864

DOI: 10.2967/jnumed.109.064238

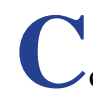

olorectal cancer (CRC) is one of the most frequently encountered malignancies in China and is associated with a high mortality rate. Currently, the treatments of choice for primary CRC are surgery, chemotherapy, and radiotherapy. However, it is not uncommon to find a difference in clinical outcomes among CRC patients even though they may have the same grade and stage of tumor. Variations in the biologic behavior of tumors are thought to cause their differing responses to the same treatment. Therefore, not only is correct staging of CRC essential, but also monitoring of the biologic behavior of tumors and response to treatment (1-3).

Among the currently available techniques, PET is increasingly used as a functional imaging routine. Unlike CT and MRI, which are anatomic imaging techniques, PET is principally a molecular modality that allows for noninvasive in vivo imaging and quantification of biologic processes such as metabolism, receptor density, cell proliferation, and uptake of therapeutic agents, thereby revealing the biologic behavior of tumors. As a result, the search for PET biomarkers is becoming increasingly popular to improve diagnosis and to monitor disease progression (4-6). 
${ }^{18}$ F-FDG is currently the most widely used PET tracer. ${ }^{18}$ F-FDG PET has become an indispensable staging modality for many types of cancer, including CRC. Nevertheless, the ability of ${ }^{18}$ F-FDG to reveal many tumor and nontumor biologic processes has been found to be unreliable. Recently, other radiotracers have been tested to complement the shortcomings of ${ }^{18}$ F-FDG. $3^{\prime}$-deoxy- $3^{\prime}-{ }^{18}$ F-fluorothymidine $\left({ }^{18} \mathrm{~F}-\mathrm{FLT}\right)$ is a pyrimidine analog and believed to be an agent for imaging cellular proliferation via the salvage pathway of DNA synthesis. The accumulation of ${ }^{18} \mathrm{~F}-\mathrm{FLT}$ is dependent on the presence of thymidine kinase-1, which is closely associated with cellular proliferation. ${ }^{18} \mathrm{~F}$-FLT has been found useful for noninvasive assessment of proliferation in several types of cancers, such as colorectal, esophageal, breast, and laryngeal $(7,8)$.

Metastasis is one of the most dreadful characteristics of malignant behavior. As indicated in the world literature, more than $50 \%$ of tumor patients die of metastases and related complications. Many factors relate to the potential of a tumor to metastasize. To better comprehend and to predict the biologic behavior of metastasis, we used both ${ }^{18} \mathrm{~F}$-FDG and ${ }^{18} \mathrm{~F}$-FLT to assess cellular uptake in $2 \mathrm{CRC}$ cell lines of different metastatic potential, and the results were analyzed along with that of animal PET and that of biomarkers for malignancy detected with Western blotting and immunocytochemistry. The 2 cell lines, SW480 and SW620, were derived from a primary lesion of a colon adenocarcinoma and from a metastatic lymph node in the same patient 1 y later. SW480 and SW620 had the same genetic background but different metastatic potential (9). Riedl et al. once reported that ${ }^{18} \mathrm{~F}-\mathrm{FDG}$ PET predicted prognosis in patients with metastatic CRC (10). Zhao et al. found that SW480 cells had a significantly higher lung metastasis rate than did SW620 cells $(11,12)$. Bearing those in mind, our intention in designing this study was to investigate whether ${ }^{18} \mathrm{~F}$-FDG and ${ }^{18} \mathrm{~F}$-FLT uptake in vitro or in vivo may be used to better predict the biologic character of metastases in CRC.

\section{MATERIALS AND METHODS}

\section{Chemicals}

RPMI 1640, Leibovitz L15 medium, and fetal bovine serum were purchased from PAA Laboratories $\mathrm{GmbH}$. All other chemicals were obtained from regular suppliers and were of reagent grade. Goat anti-integrin $\beta_{3}$, anti-heat shock protein 27 (HSP27), anti-vascular endothelial growth factor receptor 2 (VEGFR2), and anti-Ki67 polyclonal antibodies were purchased from Santa Cruz Biotechnology, Inc., and Abcam plc. A diaminobenzidine kit was obtained from Beijing Zhongshan Biotechnology Co.

\section{Cell Lines}

The human CRC cell lines SW480 and SW620 were purchased from the Institute of Cell Biology, the Chinese Academy of Sciences. According to the instructions of the provider, SW480 cells were cultured in RPMI 1640 supplemented with $10 \%$ fetal bovine serum and $1 \%$ penicillin/streptomycin at $5 \% \mathrm{CO}_{2}$ in a humidified atmosphere at $37^{\circ} \mathrm{C}$. SW620 cells were cultured in L15 medium supplemented with $10 \%$ fetal bovine serum and $1 \%$ penicillin/streptomycin, without $\mathrm{CO}_{2}$, in a humidified atmosphere at $37^{\circ} \mathrm{C}$.

\section{Animal Models}

All animal experiments were performed in accordance with the Dutch Law on Animal Experimentation and the institutional committee on animal experimentation. Fifty male BALB/C nude mice were provided by the Animal Laboratory of the Chinese Academy of Sciences. The mice were kept in sterile surroundings with a standardized light/dark cycle and access to food and water ad libitum. The mice were 6-10 wk old when used for the experiments.

Each animal bore 2 tumors via subcutaneous inoculation of $5 \times$ $10^{6}$ viable tumor cells. In 10 mice, a SW480 tumor was generated on the side of the left front leg and a SW620 tumor on the side of the right front leg. The mouse models were prepared for smallanimal PET/CT. In another 20 mice (10 in each group), SW480 and SW620 cells were injected in separate locations on the back, and the mice were continuously observed till death. Tumor size was determined by caliper measurements at least twice a week using the formula $\mathrm{V}=1 / 2(1 \times \mathrm{w} \times \mathrm{h})$, where 1 is the length, $\mathrm{w}$ the width, and $h$ the height of the tumor. ${ }^{18} \mathrm{~F}$-FDG PET/CT and ${ }^{18} \mathrm{~F}$ FLT PET/CT were performed when the tumor volume reached $100-500 \mathrm{~mm}^{3}$. In the other 20 mice, special models for metastases were generated as described below.

\section{Metastasis Assays}

Twenty BALB/C nude mice weighing 20-22 g were anesthetized with intraperitoneal injections of $1 \%$ chloral hydrate $(0.45$ $\mathrm{mg} / \mathrm{g}$ of body weight). The mice were placed supine and were handled under restrictive aseptic condition. A flank incision was made to expose the spleen. We then injected into the spleen $5 \times$ $10^{6} \mathrm{SW} 480$ or SW620 cells dissolved in phosphate-buffered saline (10 mice for each cell line). After the surgical site had been sutured, the animals were returned to their cages. The animals were killed $45 \mathrm{~d}$ after the surgery. Metastases to the liver, lungs, and peritoneal cavity were examined and counted under a microscope.

\section{PET Tracers}

${ }^{18}$ F-FDG was synthesized automatically with a conventional module used in our clinical work. ${ }^{18}$ F-FLT was synthesized according to a modified procedure originally described by $\mathrm{Oh}$ et al. (13). The entire procedure had been proven by radiopharmaceutical administration and complied with good-manufacturingpractice guidelines. Both ${ }^{18} \mathrm{~F}$-FDG and ${ }^{18} \mathrm{~F}$-FLT were pyrogen-free and qualified for clinical use, with a radiochemical purity of more than $95 \%$.

\section{Cellular Uptake of ${ }^{18} \mathrm{~F}-\mathrm{FDG}$ and ${ }^{18} \mathrm{~F}-\mathrm{FLT}$}

SW480 and SW620 cells were plated in 6-well plates at $1 \times 10^{5}$ cells per well. After $24 \mathrm{~h}, 0.05 \pm 0.01 \mathrm{MBq}$ of ${ }^{18} \mathrm{~F}-\mathrm{FDG}$ or ${ }^{18} \mathrm{~F}-$ FLT was added to the wells and incubated at $37^{\circ} \mathrm{C}$ for $30,60,90$, and $120 \mathrm{~min}$. The radioactivity added to each well was accurately measured and recorded. At the end of incubation, the cells were washed with phosphate-buffered saline twice to remove free tracer and then were detached from the well with $0.25 \%$ trypsin and accumulated in the same volume of phosphate-buffered saline. Cellular tracer uptake was assessed twice in a single-well $\gamma$-counter. The counts were averaged, and the cellular uptake was normalized to $10^{6}$ cells. 


\section{PET/CT Studies and Image Analysis}

$\mathrm{PET} / \mathrm{CT}$ images of tumor-bearing mice were obtained using a small-animal PET/CT scanner (Explore VISTA Micro-PET/CT; GE Healthcare). The mice were anesthetized using $1 \%$ chloral hydrate $(0.45 \mathrm{mg} / \mathrm{g}$ of body weight $)$ and positioned prone on the scanning table. ${ }^{18}$ F-FLT ( $n=3$ in each group) or ${ }^{18}$ F-FDG $(n=3$ in each group) was injected via the tail vein at a dose of $20 \pm 1.84$ $\mathrm{MBq}$ in $0.25 \mathrm{~mL}$ of saline. Image data were acquired for $10 \mathrm{~min}$ at $1 \mathrm{~h}$ after injection by list mode. For image reconstruction, listmode data were sorted into 3-dimensional sinograms, followed by Fourier rebinning and 2-dimensional ordered-subset expectation maximization reconstruction with 2 iterations and 50 subsets. The image pixel size was $0.385 \times 0.385 \times 0.335 \mathrm{~mm}$. For quantification of tumor uptake, ImageJ software was used on reconstructed images. Regions of interest were drawn on the tumor and the chest as background on 3 consecutive coronal slices representing the maximum tumor uptake, and the tumor-to-nontumor (background) uptake ratio (T/NT) was calculated.

\section{Immunocytochemistry Assay}

For immunocytochemistry assay, SW480 and SW620 cells were incubated on a coverslip for $24 \mathrm{~h}$ and fixed with $95 \%$ ethanol for $10 \mathrm{~min}$. After fixation, the cells were prepared with $0.5 \%(\mathrm{v} / \mathrm{v})$ $\mathrm{H}_{2} \mathrm{O}_{2}$ in methanol for $30 \mathrm{~min}$ to block endogenous peroxidase activity. The fixed cells were subjected to immunostaining using an ultrasensitive streptavidin-peroxidase technique. Briefly, the slides were incubated overnight at $4{ }^{\circ} \mathrm{C}$ with goat anti-integrin $\beta_{3}$ (SC52685), anti-HSP27 (ab2790), anti-VEGFR2 (ab3968), and antiKi67 (SC-52685) polyclonal antibody (1:100). After being washed with phosphate-buffered saline 3 times, they were treated with a biotin-conjugated second antibody before the addition of streptavidinperoxidase. Diaminobenzidine was used for color reaction.

\section{Western Blot Analysis}

Western blot analysis was performed using mouse tissue samples of SW480 and SW620 according to Zhao et al. (12). A total of $150 \mu \mathrm{g}$ of protein was analyzed by Western blotting. The antibodies were the same as described in the immunocytochemistry assay. Integrin $\beta_{3}$, HSP27, VEGFR2, and Ki67 expression were described using gray-scale analysis of Lab Works software.

\section{Statistical Analysis}

Data were statistically analyzed with the software package SPSS (version 11.5; SPSS Inc.). All data are expressed as mean \pm SD. An independent-sample $t$ test was used for analyzing tumor growth. The Fisher exact test was used for comparing differences in metastatic potential. The correlation between the results of Western blotting and radiotracer uptake or metastasis was analyzed using linear regression analysis. A $P$ value of less than 0.05 was considered statistically significant.

\section{RESULTS}

\section{Tumor Growth}

SW480 and SW620 tumors had significantly different growth rates even in the same animal. SW480 xenografts grew much faster than SW620 xenografts. It took $6.5 \pm 1.2$ $\mathrm{d}$ and $8.9 \pm 1.2 \mathrm{~d}$ for SW480 and SW620, respectively, to form macroscopic xenografts $\left(2 \mathrm{~mm}^{3}\right)(t=4.039, P=$ $0.001)$. The time for SW480 and SW620 tumors to grow to $450-500 \mathrm{~mm}^{3}$ was $24.5 \pm 3.5$ and $29.6 \pm 3.3 \mathrm{~d}$, respectively $(t=3.332, P=0.004)$. Among the 20 mice with tumors on their back, SW480-bearing mice had a survival of $59.9 \pm 6.2 \mathrm{~d}$, shorter than that of SW620-bearing mice (64.4 $\pm 6.2 \mathrm{~d}, t=2.240, P=0.038)$, without any therapeutic intervention.

\section{Potential of Metastasis}

In the current study, the metastatic potential of SW480 and SW620 was described as the incidence of metastasis to lungs and liver (Table 1). Among the 10 mice still alive after splenic injection of SW480 cells, 3 (30.0\%) had lung metastases, $4(40.0 \%)$ had liver metastases, $1(10.0 \%)$ had both lung and liver metastases, and $1(10.0 \%)$ had peritoneal lymph node metastases. In contrast, among the 9 mice still alive after splenic injection of SW620 cells, only $1(11.1 \%)$ had lung metastases, only $1(11.1 \%)$ had liver metastasis, and $3(33.3 \%)$ had higher peritoneal lymph node metastases. The incidence of metastasis to lungs and liver was significantly higher for SW480 than for SW620 $(P=0.023)$. However, there was no statistical difference in lymphatic metastases between SW480 cells and SW620 cells $(P=0.303)$.

\section{Cellular Uptake In Vitro}

After incubation for 30, 60, 90, and $120 \mathrm{~min}$, the SW480 and SW620 cells showed a time-dependent increase in ${ }^{18} \mathrm{~F}$ FDG and ${ }^{18}$ F-FLT uptake (Fig. 1). Uptake of ${ }^{18}$ F-FDG and ${ }^{18} \mathrm{~F}$-FLT was lower in SW620 cells than in SW480 cells. However, uptake of ${ }^{18} \mathrm{~F}$-FLT was higher than that of ${ }^{18} \mathrm{~F}$ FDG in both SW480 and SW620 cells.

After incubation for $60 \mathrm{~min}$, uptake of ${ }^{18} \mathrm{~F}-\mathrm{FDG}$ in SW480 and SW620 cells elevated to $1.76 \% \pm 0.87 \%$ and $1.14 \% \pm 0.38 \%$, respectively $(t=2.507, P=0.021)$. At the same time point, uptake of ${ }^{18} \mathrm{~F}$-FLT in SW480 and SW620 cells reached $5.21 \% \pm 1.60 \%$ and $2.90 \% \pm 1.82 \%$, respectively $(t=3.497, P=0.002)$.

\section{Small-Animal PET/CT Analysis}

${ }^{18}$ F-FLT PET and ${ }^{18}$ F-FDG PET images of SW480 and SW620 tumors are shown in Figure 2. ${ }^{18} \mathrm{~F}$-FLT PET showed the tumors more clearly than did ${ }^{18} \mathrm{~F}-\mathrm{FDG}$ PET. In the same animal, ${ }^{18} \mathrm{~F}$-FLT PET showed a higher radioactivity concentration in SW480 tumors than in SW620 tumors. The difference in ${ }^{18}$ F-FLT T/NT between SW480 and SW620 tumors was highly significant $(3.65 \pm 0.51$ vs. $2.22 \pm 0.42, t=6.491, P<0.001)$, whereas the ${ }^{18} \mathrm{~F}-\mathrm{FDG}$ T/NT did not differ between SW480 and SW620 tumors (2.69 \pm 0.98 vs. $3.09 \pm 1.26, t=0.657, P=0.524)$.

TABLE 1. In Vivo Experiment on Metastases in 20 Nude Mice

\begin{tabular}{lcc}
\hline & $\begin{array}{c}\text { Liver and lung } \\
\text { metastases }\end{array}$ & $\begin{array}{c}\text { Peritoneal lymph } \\
\text { node metastases }\end{array}$ \\
SW line & $8 / 10^{*}$ & $1 / 10$ \\
SW620 & $2 / 9^{*}$ & $3 / 9$ \\
& \\
\hline & \\
\hline
\end{tabular}



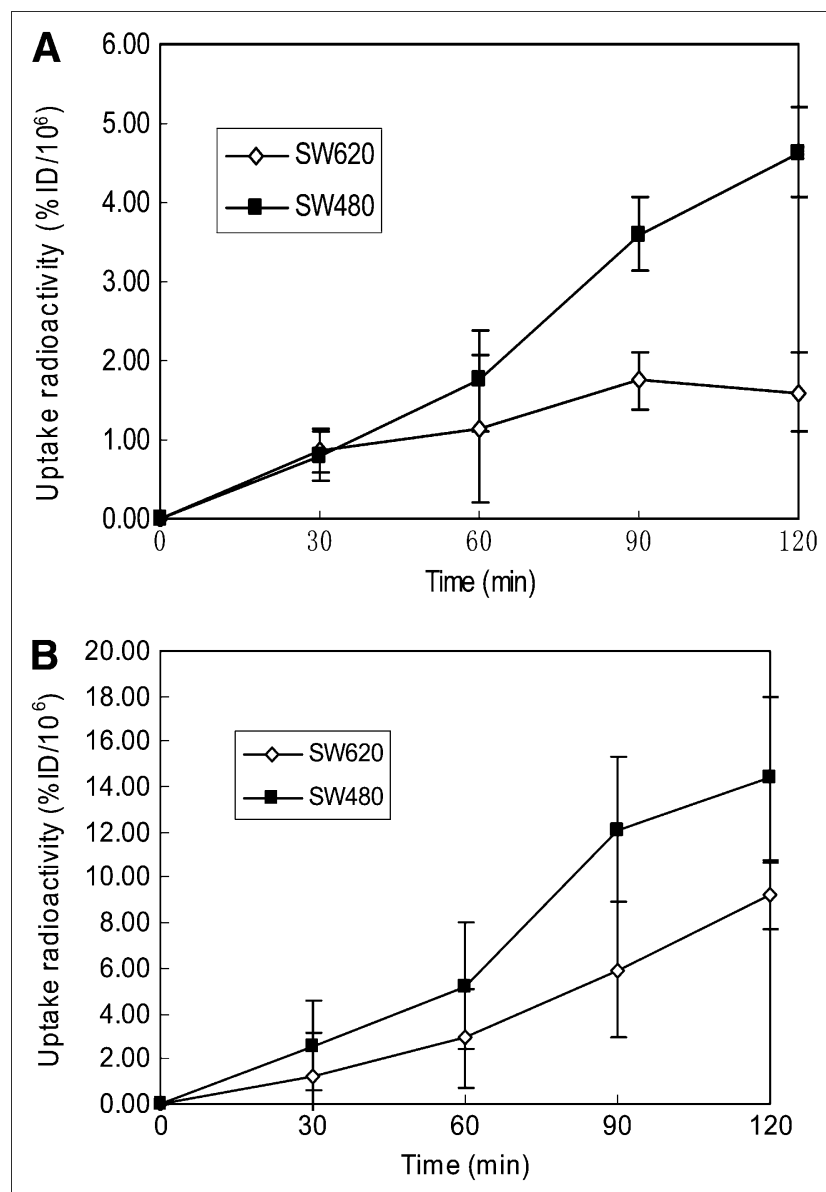

FIGURE 1. Cellular uptake of ${ }^{18} \mathrm{~F}-\mathrm{FDG}$ and ${ }^{18} \mathrm{~F}-\mathrm{FLT}$ in vitro. Data are expressed as percentage injected dose (\%ID) of ${ }^{18} \mathrm{~F}-\mathrm{FDG}(\mathrm{A})$ or ${ }^{18} \mathrm{~F}-\mathrm{FLT}$ (B) per $10^{6}$ cells. Error bar depicts SD $(n=10-15)$. After incubation for $60 \mathrm{~min}$, uptake of ${ }^{18} \mathrm{~F}-\mathrm{FDG}$ was different between SW480 and SW620 cells $(t=-2.507$, $P=0.021$ ). At same time point, uptake of ${ }^{18} \mathrm{~F}-\mathrm{FLT}$ was significantly different between SW480 and SW620 cells $(t=$ 3.497, $P=0.002$ ).

\section{Immunocytochemistry and Western Blot Analysis}

HSP27, integrin $\beta_{3}$, VEGFR2, and Ki67 were overexpressed in SW480 and SW620 cells, as shown in Figure 3. SW480 cells showed more intense brown staining of HSP27 and integrin $\beta_{3}$ in the cytoplasm and nucleus. In contrast, VEGFR2 and Ki67 staining was weaker in SW480 cells than in SW620 cells. Ki67 stained the nucleus more intensely in SW620 cells than in SW480 cells.

The expression of HSP27, integrin $\beta_{3}$, VEGFR2, and Ki67 was examined by Western blotting. As shown in Figure 4, expression of HSP27 and integrin $\beta_{3}$ was greater in SW480 tumors $(42.86 \% \pm 5.15 \%$ vs. $9.61 \% \pm 3.20 \%)$ than in SW620 tumors $(10.10 \% \pm 3.50 \%$ vs. $8.43 \% \pm$ $1.85 \%)$. Expression of VEGFR2 and $\mathrm{Ki} 67$ was less pronounced in SW480 tumors $(9.00 \% \pm 2.38 \%$ vs. $6.5 \% \pm$ $1.25 \%)$ than in SW620 tumors $(19.96 \% \pm 4.20 \%$ vs. $25.33 \% \pm 5.59 \%)$.

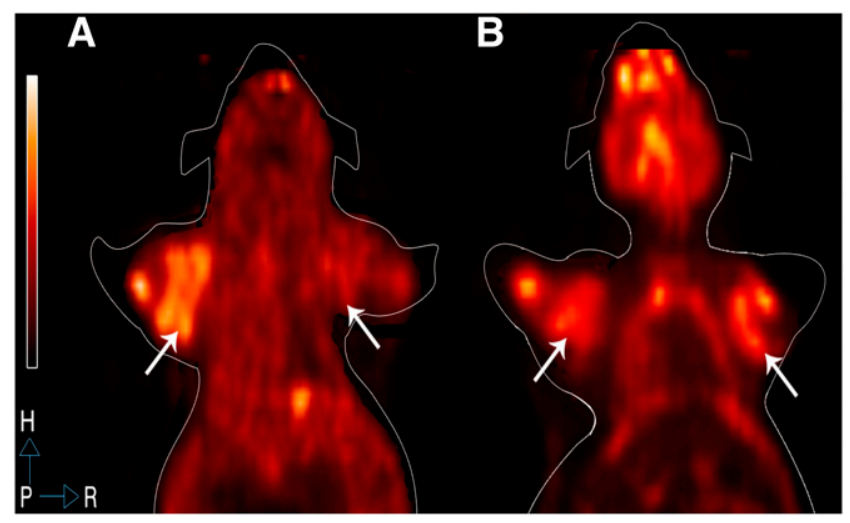

FIGURE 2. ${ }^{18} \mathrm{~F}-\mathrm{FLT}$ PET and ${ }^{18} \mathrm{~F}-\mathrm{FDG}$ PET images of SW480 and SW620 tumors. (A) ${ }^{18} \mathrm{~F}$-FLT PET image shows that uptake of ${ }^{18} \mathrm{~F}-\mathrm{FLT}$ in SW480 tumors (left) was markedly stronger than that in SW620 tumors (right) $(t=6.491, P<$ 0.001 ). (B) ${ }^{18} \mathrm{~F}-\mathrm{FDG}$ PET image shows that uptake of ${ }^{18} \mathrm{~F}-$ FDG in SW480 tumors (left) was weaker than that in SW620 tumors (right), with no significant difference $(t=0.657, P=$ 0.524 ) ( $n=3$ per group).

\section{Linear Regression Analysis}

No correlation was found between ${ }^{18} \mathrm{~F}-\mathrm{FDG}$ uptake and ${ }^{18}$ F-FLT uptake in tumors $(r=-0.067, P=0.45)$. Cellular uptake of ${ }^{18} \mathrm{~F}$-FLT in vitro correlated with tumor uptake in vivo $(r=0.553, P=0.009)$. A significantly correlation was found between ${ }^{18} \mathrm{~F}$-FLT uptake in tumors and the number of metastases to the lung and liver $(r=0.763, P=0.005)$, but no such correlation was found for ${ }^{18}$ F-FDG $(r=-0.111$, $P=0.388$ ) (Fig. 5). A significant correlation existed between HSP27 expression and metastatic potential $(r=$ $0.894, P=0.008)$, whereas the correlation was not significant between integrin $\beta_{3}$ expression and metastasis $(r=$ $0.635, P=0.088)$. A significant correlation was observed between ${ }^{18}$ F-FLT T/NT and HSP27 expression $(r=0.924$, $P=0.004)$ and between ${ }^{18} \mathrm{~F}-\mathrm{FLT}$ T/NT and integrin $\beta_{3}$ expression ( $r=0.813, P=0.025$ ) (Fig. 6). A significantly negative correlation was observed between ${ }^{18} \mathrm{~F}$-FDG uptake and the survival time of tumor-bearing mice $(r=-0.500$, $P=0.017)$, and a positive correlation was observed between ${ }^{18} \mathrm{~F}$-FDG uptake and lymph node metastases $(r=$ $0.825, P=0.003)$. However, ${ }^{18} \mathrm{~F}$-FLT uptake in tumors was not related to survival ( $r=0.262, P=0.182$ ) (Fig. 7).

\section{DISCUSSION}

${ }^{18}$ F-FDG PET and ${ }^{18}$ F-FLT PET are clinically used for the diagnosis, staging, and restaging of a wide variety of tumors. Recently, the importance of multimodality imaging for characterization of tumors has become apparent. It has been well understood that tumor biologic behaviors such as proliferation, angiogenesis, abnormal expression of protein, invasion, and metastasis are profoundly influenced by many factors and by interactions among those factors. It has also been recognized that the complexity of tumor behavior is beyond the ability of any single radiotracer, or radioactive 


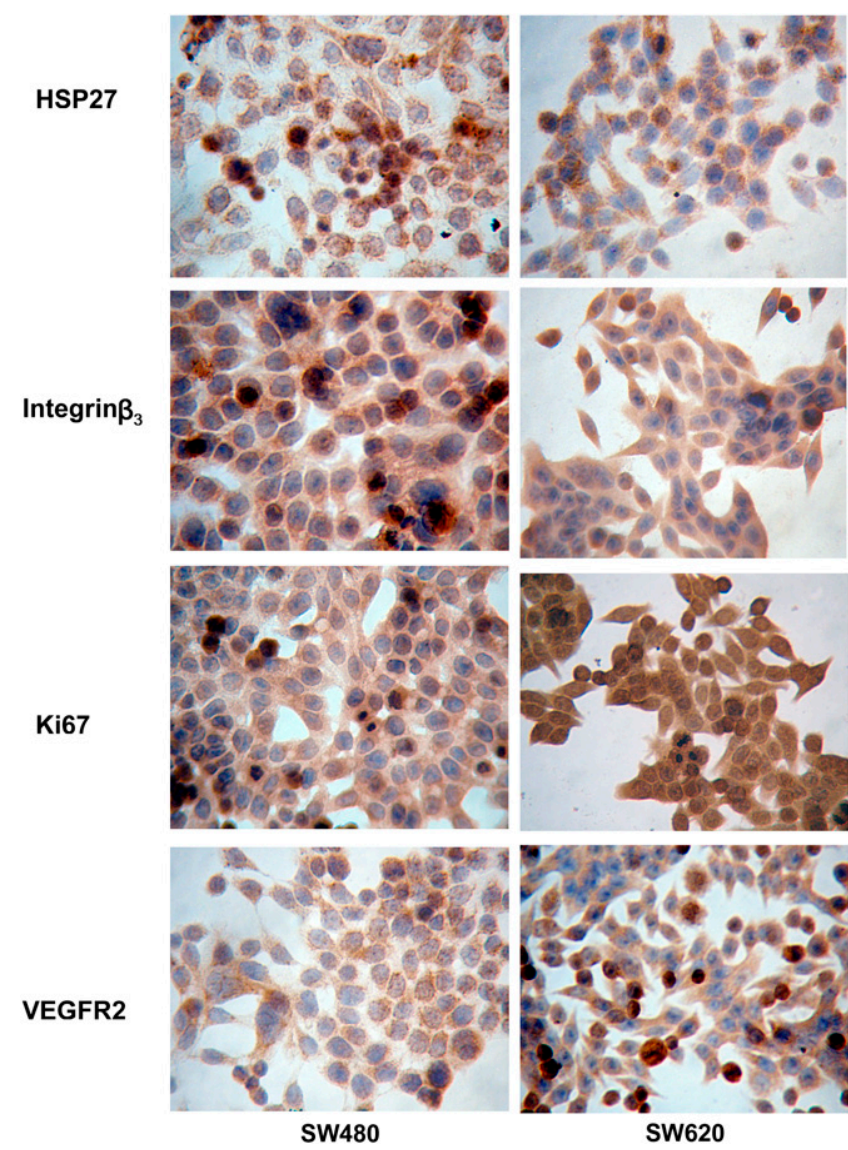

FIGURE 3. Expression of HSP27, integrin $\beta_{3}$, Ki67, and VEGFR2 in SW480 (left column) and SW620 (right column) cell lines by immunocytochemistry detection $(\times 400)$. HSP27 expression in cytoplasm and nucleus was significantly higher in SW480 than in SW620. Integrin $\beta_{3}$ expression in cytoplasm and cytomembrane was higher in SW480 than in SW620. Ki67 expression in nucleus was higher in SW620 than in SW480. VEGFR2 expression in cytoplasm and cytomembrane was higher in SW620 than in SW480.

molecular probe, to reveal. Several tests have shown that ${ }^{18} \mathrm{~F}-\mathrm{FDG}$ PET measures the glucose metabolism and certain genetic alterations of tumors $(14,15)$. The high uptake of ${ }^{18} \mathrm{~F}-\mathrm{FDG}$ in tumors was proven of value in assessing prognosis. Although several other research groups have claimed that ${ }^{18} \mathrm{~F}$-FLT is a more proliferation-specific tracer $(10,16)$, the present study used both ${ }^{18} \mathrm{~F}-\mathrm{FDG}$ and ${ }^{18} \mathrm{~F}$ FLT - each representing different biologic characters-as potential tools for monitoring the biologic behavior of human CRC lines.

Metastasis is the neoplastic process responsible for most deaths from cancer. Approximately $50 \%$ of cancer patients die within $5 \mathrm{y}$ of diagnosis because of cancer-related problems, mostly attributable to metastatic spread to nearby critical organs or to distant sites. Even after potentially curative surgery, metastases subsequently develop in more than $30 \%$ of patients with CRC. The liver is involved in more than $70 \%$ of patients, and $40 \%-50 \%$ of those with

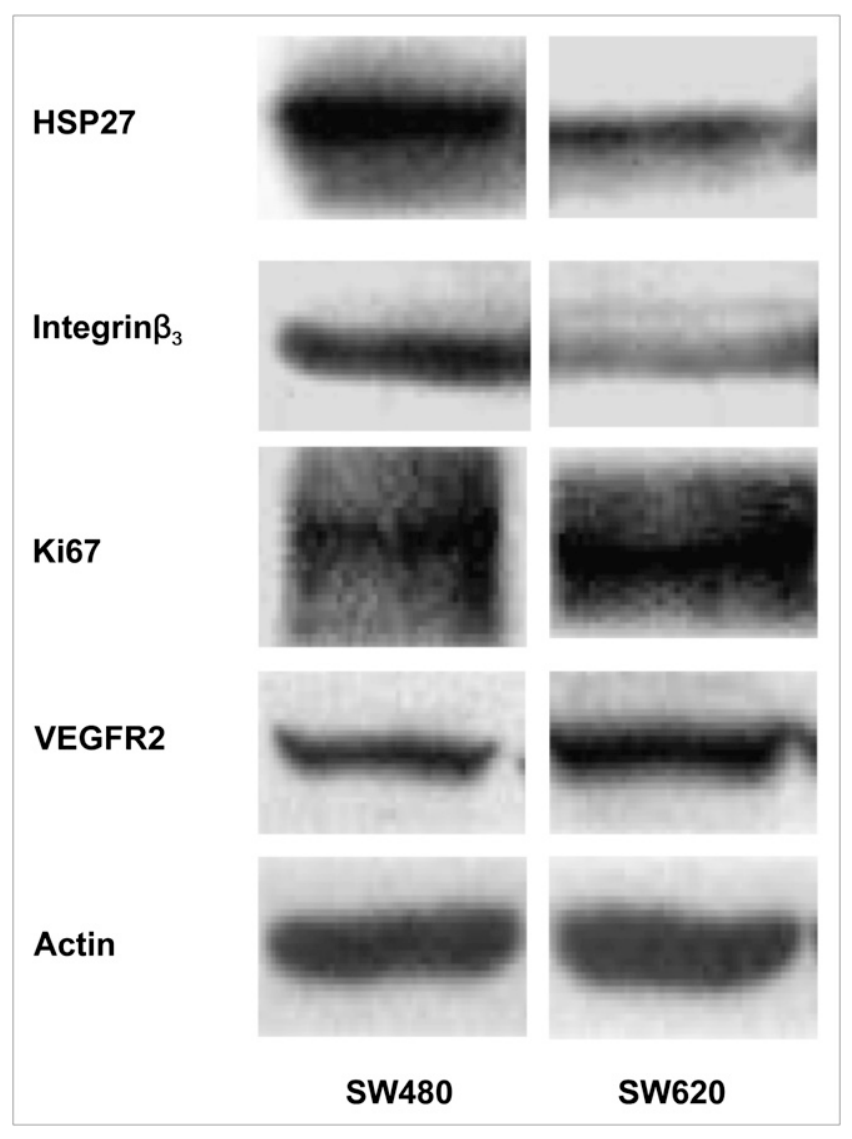

FIGURE 4. Western blot analysis of HSP27, integrin $\beta_{3}$, Ki67, VEGFR2, and actin expression. Expression of HSP27 and integrin $\beta_{3}$ was greater in SW480 tumors than in SW620 tumors. Expression of Ki67 and VEGFR2 was less in SW480 tumors than in SW620 tumors. Actin antibody is loading control.

liver metastases have metastatic colonization in other organs. SW480 and SW620 cells derived from the same patient with the same genetic background provide a good model for researching different metastatic characteristics (17). Zhao et al. found that SW480 cells had a significantly higher lung metastasis rate than SW620 cells (11). Kubens et al. also proved that SW480 cells had a higher migration capacity than SW620 cells (18). But little has been known about whether the difference in metastatic potential can be revealed or predicted in vivo by PET and other imaging techniques. The current study was thus designed to verify the ability of the 2 radiotracers to reveal the metastatic character of the 2 cell lines. Clinical outcome and the molecular biomarkers served as a gold standard and were used as the foundation for comparative analysis.

The study showed that the biologic behavior of SW480 tumors was significantly different from that of SW620 tumors. The subcutaneous SW480 xenografts grew more quickly, under similar conditions in the same animal, than did the SW620 xenografts. The survival time of SW480bearing mice was shorter significantly than that of 

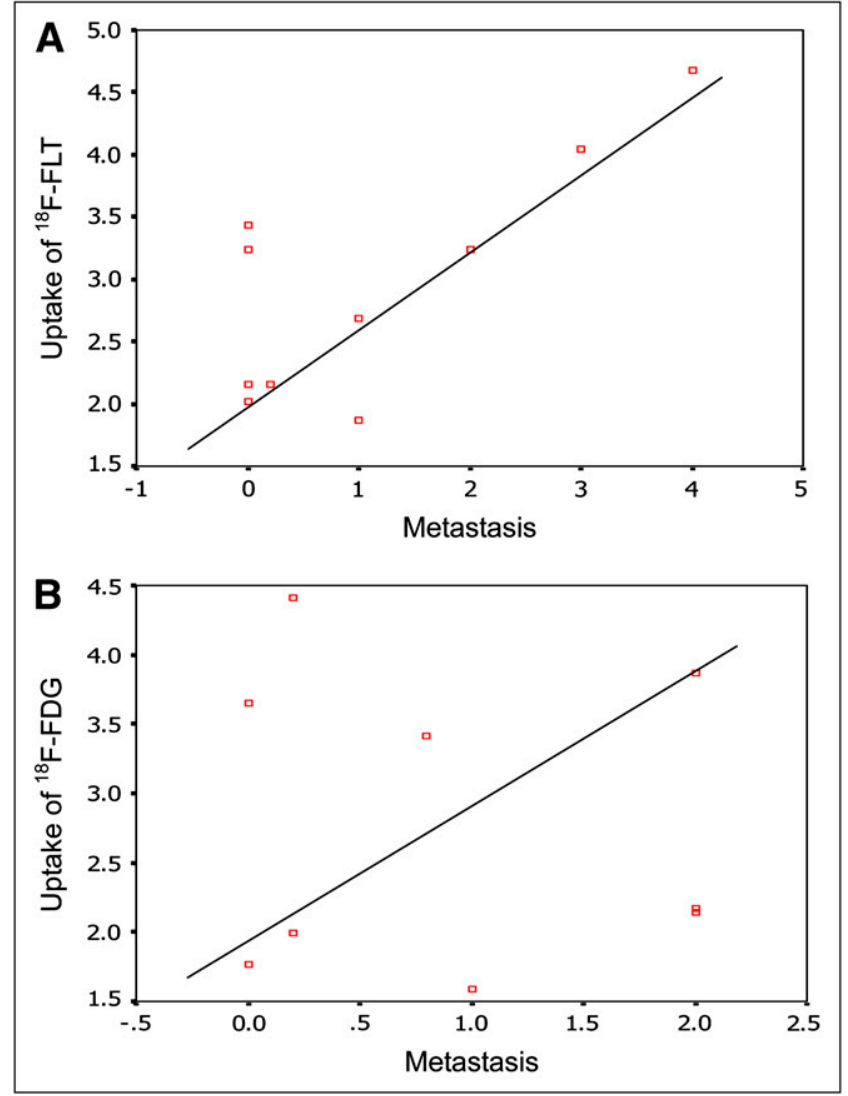

FIGURE 5. (A) Linear regression analysis shows significant correlation between metastatic activity and uptake of ${ }^{18} \mathrm{~F}$ FLT PET in CRC ( $r=0.763, P=0.005)$. (B) No significant correlation is seen between metastatic activity and uptake of ${ }^{18} \mathrm{~F}-\mathrm{FDG}$ PET in CRC $(r=-0.111, P=0.388)$.

SW620-bearing mice. The SW480 models had a significantly higher rate of lung and liver metastasis but a lower rate of lymph node metastasis than the SW620 models. Those results were in keeping with a previous description of the 2 cell lines indicating that cells with the same genetic origin might indeed behave differently (11).

Uptake of ${ }^{18} \mathrm{~F}$-FDG and ${ }^{18} \mathrm{~F}$-FLT was proven to be higher in SW480 cells than in SW620 cells, with uptake of ${ }^{18} \mathrm{~F}-$ FLT being higher than that of ${ }^{18} \mathrm{~F}-\mathrm{FDG}$ in both cell lines. By in vivo small-animal PET, ${ }^{18} \mathrm{~F}-\mathrm{FLT}$ T/NT showed a statistically significant difference between SW480 and SW620 tumors, as was in accordance with the results of the in vitro cellular uptake test. However, contrary to the results of the in vitro ${ }^{18} \mathrm{~F}-\mathrm{FDG}$ uptake test, no significant difference in ${ }^{18} \mathrm{~F}$-FDG T/NT was found between SW480 and SW620 tumors. The differences between the 2 tracers were considered due to the effects of microenvironment or blood circulation or to differences in metabolism or the pathways involved.

Tumor biomarkers, a big family with ever-increasing numbers, play a major role in monitoring tumor characteristics; exploring possible mechanisms of antitherapy; and evaluating and suggesting new therapeutic approaches.
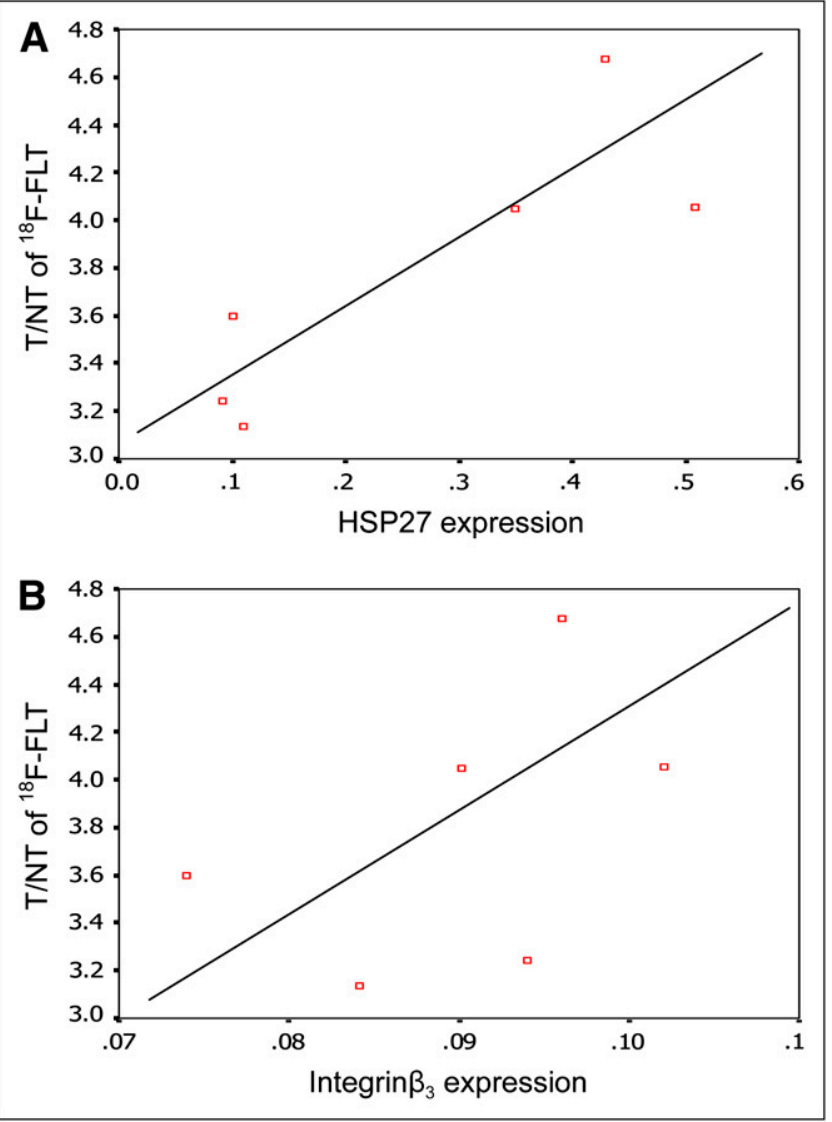

FIGURE 6. Linear regression analysis shows significant correlation between HSP27 expression and T/NT for ${ }^{18} \mathrm{~F}-\mathrm{FLT}$ PET in CRC $(r=0.924, P=0.004)(A)$ and between integrin $\beta_{3}$ expression and T/NT for ${ }^{18}$ F-FLT PET in CRC $(r=0.813$, $P=0.025)(B)$.

Many different proteins are expressed in SW480 and SW620 cell lines (19). In the current study, we selected 4 tumor biomarkers-HSP27, integrin $\beta_{3}$, VEGFR2, and Ki67-known to relate to tumor differentiation, invasion, angiogenesis, and proliferation, respectively (20-23).

Heat shock proteins are overexpressed in a wide range of human cancers and are implicated in several characteristics of tumor biology, including differentiation, invasion, and immunorecognition. HSP27 is a member of the heat shock protein family. Numerous clinical studies indicate a positive correlation between the expression of HSP27 and the invasiveness or metastatic capacity of tumors. The mechanisms of invasion and metastasis for HSP27 include the increased ability of tumor cells to survive in the bloodstream because of death-inhibitory properties $(24,25)$. Integrin $\beta_{3}$ is believed to relate to angiogenesis and is also overexpressed in many human cancers. In our study, we found that HSP27 and integrin $\beta_{3}$ overexpression in SW480 was related to high metastatic potential of the tumor, and the overexpression correlated well with uptake of ${ }^{18} \mathrm{~F}$-FLT both in vitro and in vivo. Although uptake of ${ }^{18} \mathrm{~F}$-FLT was lower in SW620 tumors or tumor cells, so was HSP27 and 

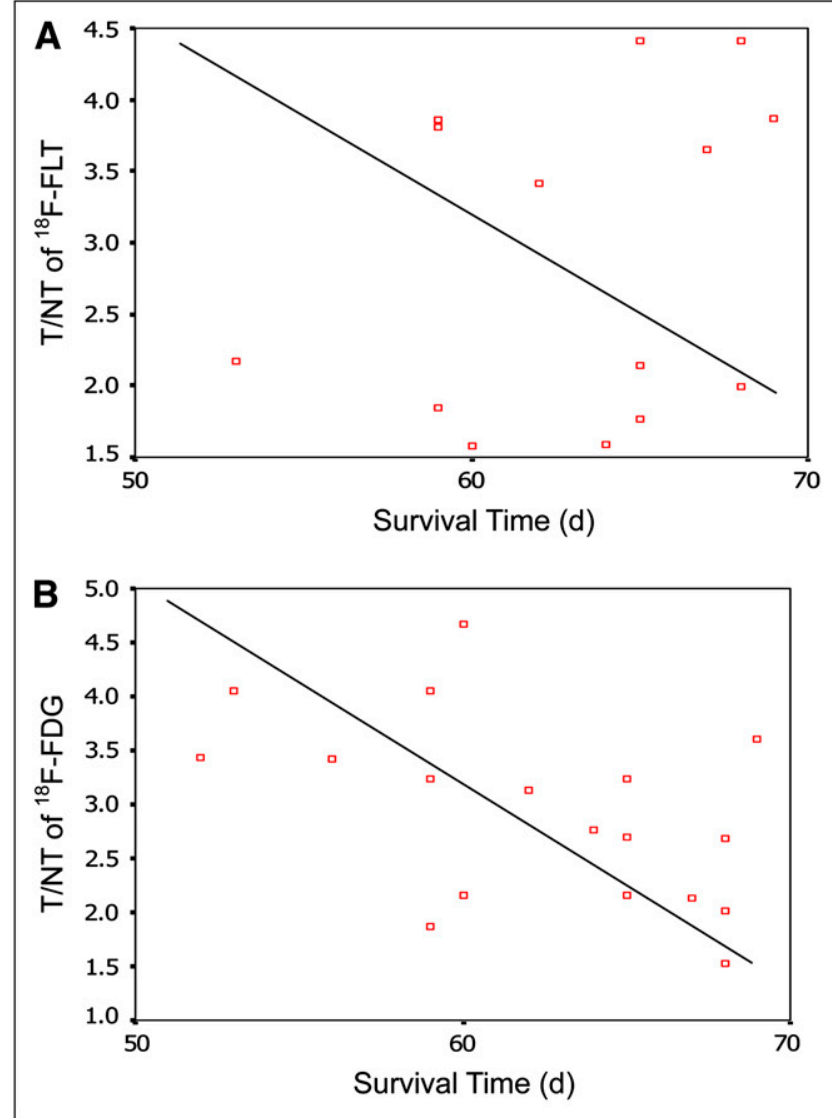

FIGURE 7. (A) Linear regression analysis shows no significant correlation between survival of tumor-bearing mice and T/NT for ${ }^{18} \mathrm{~F}-\mathrm{FLT}$ PET $(r=0.262, P=0.182)$. (B) Significant correlation is seen between survival of tumorbearing mice and T/NT for ${ }^{18} \mathrm{~F}-\mathrm{FDG}$ PET $(r=-0.500, P=$ 0.017).

integrin $\beta_{3}$. No correlation was observed between metastasis and ${ }^{18}$ F-FDG uptake, even though cellular uptake in vitro had shown a difference in ${ }^{18} \mathrm{~F}-\mathrm{FDG}$ between SW480 and SW620.

Uptake of ${ }^{18} \mathrm{~F}$-FLT in tumors did not positively correlate with Ki67 or VEGFR $_{2}$ expression levels. However, VEGFR2 and Ki67 were overexpressed in SW620 cells, and lymph node metastasis correlated with Ki67 and VEGFR $_{2}$ in tumors. One might just assume that metastasis via hematologic and lymphatic means might have different pathways. Though Ki67 is widely recognized as a biomarker for cellular proliferation, someone suggested that thymidine kinase- 1 is a more direct and important proliferation indicator than Ki67 $(26,27)$. Detecting thymidine kinase-1 activity would more accurately reveal proliferative ability.

Our results showed that HSP27 and integrin $\beta_{3}$ were overexpressed in SW480, reflecting a higher rate of metastasis to lung and liver. The metastatic ability could be demonstrated by higher ${ }^{18} \mathrm{~F}$-FLT uptake. However, VEGFR2 and Ki67 were overexpressed in SW620 cells, which could be reflected by ${ }^{18} \mathrm{~F}-\mathrm{FDG}$ uptake but not by ${ }^{18} \mathrm{~F}$-FLT uptake. ${ }^{18} \mathrm{~F}-\mathrm{FDG}$ uptake was slightly higher in SW620 tumors than in SW480 tumors, but the difference did not reach statistical significance. Although ${ }^{18} \mathrm{~F}-\mathrm{FDG}$ could not suitably predict lung and liver metastasis, our study showed that ${ }^{18} \mathrm{~F}$-FDG T/NT correlated negatively with the survival time of tumor-bearing mice and with the incidence of lymphatic metastasis of SW620. ${ }^{18}$ F-FLT, on the other hand, had no such power to indicate survival or lymphatic metastasis in SW480- and SW620-bearing mice.

Though the study population was small, it was interesting to find that ${ }^{18} \mathrm{~F}$-FDG uptake and ${ }^{18} \mathrm{~F}$-FLT uptake in SW480 and SW620 cells and tumors were so different. It was even more interesting to find that the differences in ${ }^{18} \mathrm{~F}-\mathrm{FLT}$ and ${ }^{18} \mathrm{~F}$-FDG uptake eventually reflected differences in tumor biomarker expression and, further, the metastatic potential of the 2 tumor cell lines. It thus seemed feasible to postulate that high uptake of ${ }^{18} \mathrm{~F}$-FLT could positively predict a higher incidence of metastasis, whereas high uptake of ${ }^{18} \mathrm{~F}-\mathrm{FDG}$ was possibly predictive of lymphatic metastases and prognosis. Though the mechanism is not clear, our results suggested that the use of both tracers together would be critical to revealing more about the biologic character of the tumor. Any single tracer, ${ }^{18} \mathrm{~F}$-FLT or ${ }^{18} \mathrm{~F}-\mathrm{FDG}$, may not be adequate in precisely predicting the biologic behavior of tumors. Predicting biologic behavior would be helpful for individuation of tumor treatment in clinical practice. Additional studies are ongoing in more animals, and clinical studies are needed to confirm the relationship between dual-tracer PET and the biologic character of CRC.

\section{CONCLUSION}

High uptake of ${ }^{18} \mathrm{~F}-\mathrm{FDG}$ and ${ }^{18} \mathrm{~F}$-FLT may reflect poorer survival and a higher metastatic potential for CRC in mice. Combining ${ }^{18} \mathrm{~F}$-FDG with ${ }^{18} \mathrm{~F}$-FLT PET would be helpful in better predicting the biologic behavior of CRC.

\section{ACKNOWLEDGMENTS}

This work was supported by grants from the China Postdoctoral Science Foundation (20070420569) and the National Natural Science Foundation of China (3077607). We thank Gao Fei and Zou Ke for their expert assistance with training on small-animal PET/CT, and we thank $\mathrm{Li}$ Xuegang and Zhang Xiaojun for help with synthesizing ${ }^{18} \mathrm{~F}-\mathrm{FLT}$ and ${ }^{18} \mathrm{~F}-\mathrm{FDG}$. Niu Baolong is acknowledged for help with radiation dose calculations, and Liu Ziwen and Yang Zhigang for help with Western blotting and immunocytochemistry assays.

\section{REFERENCES}

1. Mohiuddin M, Marks J, Marks G. Management of rectal cancer: short- vs. long-course preoperative radiation. Int J Radiat Oncol Biol Phys. 2008;72: 636-643. 
2. Mathis KL, Nelson H, Pemberton JH, Haddock MG, Gunderson LL. Unresectable colorectal cancer can be cured with multimodality therapy. Ann Surg. 2008;248:592-598.

3. Low G, Tho LM, Leen E, et al. The role of imaging in the pre-operative staging and post-operative follow-up of rectal cancer. Surgeon. 2008;6:222-231.

4. Stephen RM, Gillies RJ. Promise and progress for functional and molecular imaging of response to targeted therapies. Pharm Res. 2007;24:1172-1185.

5. Hicks RJ. The role of PET in monitoring therapy. Cancer Imaging. 2005;5:51-57.

6. Rajendran JG, Mankoff DA. Beyond detection: novel applications for PET imaging to guide cancer therapy. J Nucl Med. 2007;48:855-856.

7. Yamamoto Y, Nishiyama Y, Kimura N, et al. Comparison of ${ }^{18}$ F-FLT PET and ${ }^{18} \mathrm{~F}$-FDG PET for preoperative staging in non-small cell lung cancer. Eur J Nucl Med Mol Imaging. 2008;35:236-245.

8. Yamamoto Y, Nishiyama Y, Ishikawa S, et al. Correlation of ${ }^{18} \mathrm{~F}-\mathrm{FLT}$ and ${ }^{18} \mathrm{~F}$ FDG uptake on PET with Ki-67 immunohistochemistry in non-small cell lung cancer. Eur J Nucl Med Mol Imaging. 2007;34:1610-1616.

9. Duranton B, Holl V, Schneider Y, et al. Polyamine metabolism in primary human colon adenocarcinoma cells (SW480) and their lymph node metastatic derivatives (SW620). Amino Acids. 2003;24:63-72.

10. Riedl CC, Akhurst T, Larson S, et al. ${ }^{18}$ F-FDG PET scanning correlates with tissue markers of poor prognosis and predicts mortality for patients after liver resection for colorectal metastases. J Nucl Med. 2007;48:771-775.

11. Zhao L, Liu L, Wang S, Zhang YF, Yu L, Ding YQ. Differential proteomic analysis of human colorectal carcinoma cell lines metastasis-associated proteins. J Cancer Res Clin Oncol. 2007;133:771-782.

12. Zhao L, Li ZG, Ding YQ. Expression of HSP27 in colorectal carcinoma and its relationship with lymphatic metastasis [in Chinese]. Nan Fang Yi Ke Da Xue Xue Bao. 2008;28:41-44.

13. Oh SJ, Mosdzianowski C, Chi DY, et al. Fully automated synthesis system of 3'-deoxy-3'-[ ${ }^{18}$ F] fluorothymidine. Nucl Med Biol. 2004;31:803-809.

14. Cermik T, Houseni M, Alavi A. Implications of standardized uptake value measurements of the primary lesions in proven cases of breast carcinoma with different degree of disease burden at diagnosis: does 2-deoxy-2-[F-18] fluoro-D-glucose-positron emission tomography predict tumor biology? Mol Imaging Biol. 2008;10:62-66.

15. Roels S, Slagmolen P, Nuyts J, et al. Biological image-guided radiotherapy in rectal cancer: is there a role for FMISO or FLT, next to FDG? Acta Oncol. 2008;47:1237-1248.
16. Been LB, Suurmeijer AJ, Cobben DC, Jager PL, Hoekstra HJ, Elsinga PH. $\left[{ }^{18}\right.$ F]FLT-PET in oncology: current status and opportunities. Eur J Nucl Med Mol Imaging. 2004;31:1659-1672.

17. Schlüter K, Gassmann P, Enns A, et al. Organ-specific metastatic tumor cell adhesion and extravasation of colon carcinoma cells with different metastatic potential. Am J Pathol. 2006;169:1064-1073.

18. Kubens BS, Zänker KS. Differences in the migration capacity of primary human colon carcinoma cells (SW480) and their lymph node metastatic derivatives (SW620). Cancer Lett. 1998;131:55-64.

19. Katayama M, Nakano H, Ishiuchi A, et al. Protein pattern difference in the colon cancer cell lines examined by two-dimensional differential in-gel electrophoresis and mass spectrometry. Surg Today. 2006;36:1085-1093.

20. Robertson JH, Iga AM, Sales KM, Winslet MC, Seifalian AM. Integrins: a method of early intervention in the treatment of colorectal liver metastases. Curr Pharm Des. 2008;14:296-305.

21. Kim EA, Gershtein ES, Vysotskaya IV, Kushlinskii NE. Expression of VEGF and VEGFR2 in tumors during neoadjuvant therapy of patients with breast cancer. Bull Exp Biol Med. 2008;145:245-248.

22. Kushlinsky NE, Trapeznikova MF, Gershtein ES, Glibin PA, Kazantceva IA, Kilichbekov MB. Vascular endothelial growth factor and its type 2 receptor in tumors and serum of patients with renal cancer. Bull Exp Biol Med. 2008; 145:744-747.

23. Glaessgen A, Jonmarker S, Lindberg A, et al. Heat shock proteins 27,60 and 70 as prognostic markers of prostate cancer. APMIS. 2008;116:888-895.

24. Ciocca DR, Calderwood SK. Heat shock proteins in cancer: diagnostic, prognostic, predictive, and treatment implications. Cell Stress Chaperones. 2005;10:86-103.

25. Calderwood SK, Khaleque MA, Sawyer DB, Ciocca DR. Heat shock proteins in cancer: chaperones of tumorigenesis. Trends Biochem Sci. 2006;31:164172.

26. Salskov A, Tammisetti VS, Grierson J, Vesselle H. FLT: measuring tumor cell proliferation in vivo with positron emission tomography and $3^{\prime}$-deoxy- $3^{\prime}$ - $\left[{ }^{18} \mathrm{~F}\right]$ fluoro-thymidine. Semin Nucl Med. 2007;37:429-439.

27. Francis DL, Visvikis D, Costa DC, et al. Potential impact of $\left[{ }^{18} \mathrm{~F}\right] 3^{\prime}$-deoxy-3' fluorothymidine versus $\left[{ }^{18} \mathrm{~F}\right]$ fluoro-2-deoxy-D-glucose in positron emission tomography for colorectal cancer. Eur J Nucl Med Mol Imaging. 2003;30: 988-994. 